\title{
On Gale and braxial polytopes
}

\author{
Margaret M. Bayer* \\ Department of Mathematics \\ University of Kansas \\ Lawrence KS 66045-7523 USA \\ Tibor Bisztriczky ${ }^{\dagger}$ \\ Department of Mathematics and Statistics \\ University of Calgary \\ Calgary, Alberta, T2N 1N4 Canada
}

September 2006

\begin{abstract}
Cyclic polytopes are characterized as simplicial polytopes satisfying Gale's evenness condition (a combinatorial condition on facets relative to a fixed ordering of the vertices). Periodically-cyclic polytopes are polytopes for which certain subpolytopes are cyclic. Bisztriczky discovered a class of periodically-cyclic polytopes that also satisfy Gale's evenness condition. The faces of these polytopes are braxtopes, a certain class of nonsimplicial polytopes studied by the authors. In this paper we prove that the periodically-cyclic Gale polytopes of Bisztriczky are exactly the polytopes that satisfy Gale's evenness condition and are braxial (all faces are braxtopes). The existence of other periodicallycyclic Gale polytopes is open.
\end{abstract}

\section{Introduction}

We recall that cyclic polytopes have a totally ordered set of vertices (vertex array) that satisfies Gale's Evenness Condition and yields a complete description of their facet structure. One seeks to generalize this class of polytopes due to their important combinatorial properties and to their many

\footnotetext{
* Supported in part by a grant from the University of Kansas General Research Fund

${ }^{\dagger}$ Supported in part by a Natural Sciences and Engineering Research Council of Canada Discovery Grant
} 
applications in various branches of mathematics and science. Of specific significance are generalizations that are nonsimplicial and that exhibit constructions other than products, pyramids, prisms and so forth. Bicyclic 4-polytopes, ordinary $d$-polytopes and certain periodically-cyclic Gale $d$ -

polytopes are examples of such generalizations. It is noteworthy that these are also polytopes with explicit facet structures.

Our present interest is characterizations of these polytopes that do not invoke their constructions or facet structures. For example: cyclic polytopes may be characterized as Gale and simplicial polytopes or as neighbourly polytopes with the same number of universal edges as vertices or as polytopes that have only cyclic subpolytopes. With the observation that multiplices are generalizations of simplices, ordinary polytopes may be characterized as Gale and multiplicial polytopes. With the knowledge that braxtopes are also generalizations of simplices and that they were discovered as facets of certain periodically-cyclic Gale polytopes, it is natural to ask if there is a characterization of periodically-cyclic Gale polytopes as polytopes that are Gale and braxial. In the following, we determine that all Gale and braxial $d$-polytopes are periodically-cyclic for $d \geq 5$.

\section{Definitions and background}

Let $Y$ be a set of points in $\mathbf{R}^{d}, d \geq 1$. Then $[Y]$ and $\langle Y\rangle$ denote, respectively, the convex hull and the affine hull of $Y$. If $Y=\left\{y_{1}, y_{2}, \ldots, y_{s}\right\}$ is finite, we set $\left[y_{1}, y_{2}, \ldots, y_{s}\right]=[Y]$ and $\left\langle y_{1}, y_{2}, \ldots, y_{s}\right\rangle=\langle Y\rangle$

Let $X=\left\{x_{0}, x_{1}, \ldots, x_{n}\right\}$ be a totally ordered set of $n+1$ points in $\mathbf{R}^{d}$ with $x_{i}<x_{j}$ if and only if $i<j$. We say that $x_{i}$ and $x_{i+1}$ are successive points, and if $x_{i}<x_{j}<x_{k}$ then $x_{j}$ separates, or is between, $x_{i}$ and $x_{k}$. Let $Y \subset X$. Then $Y$ is a Gale subset of $X$ if any two points of $X \backslash Y$ are separated by an even number of points of $Y$. Finally, $X$ is a paired set if it is the union of mutually disjoint $\left\{x_{i}, x_{i+1}\right\}$. As a rule, $S_{m}$ denotes a paired set of $m$ points with $S_{0}$ denoting the empty set.

Let $P \subset \mathbf{R}^{d}$ be a (convex) $d$-polytope. For $-1 \leq i \leq d$, let $\mathcal{F}_{i}(P)$ denote the set of $i$-dimensional faces of $P$ with $f_{i}(P)=\left|\mathcal{F}_{i}(P)\right|$. For convenience, let

$$
\mathcal{V}(P)=\mathcal{F}_{0}(P), \mathcal{E}(P)=\mathcal{F}_{1}(P), \text { and } \mathcal{F}(P)=\mathcal{F}_{d-1}(P) .
$$

We assume familiarity with the basic definitions and concepts concerning polytopes (see [9, 12]), and we cite two results necessary for our presentation from [9] and [10, respectively. 
Lemma 1 Let $P^{\prime}$ and $P$ be d-polytopes in $\mathbf{R}^{d}$ such that $P=\left[P^{\prime}, x\right]$ for some point $x \in \mathbf{R}^{d} \backslash P^{\prime}$. Let $G$ be a face of $P^{\prime}$ and $\mathcal{F}\left(G, P^{\prime}\right)=\left\{F \in \mathcal{F}\left(P^{\prime}\right) \mid G \subseteq\right.$ $F\}$. Then

a. $G$ is a face of $P$ if and only if $x$ is beneath some $F \in \mathcal{F}\left(G, P^{\prime}\right)$;

b. $[G, x]$ is a face of $P$ if and only if either $x \in\langle G\rangle$ or $x$ is beneath $F^{\prime}$ and beyond $F^{\prime \prime}$ for some $F^{\prime}$ and $F^{\prime \prime}$ in $\mathcal{F}\left(G, P^{\prime}\right)$; and

c. if $G$ is a face of $P$ and $[G, x]$ is not a face of $P$, then $x$ is not beyond any $F \in \mathcal{F}\left(G, P^{\prime}\right)$.

Lemma 2 If the facet system of one d-polytope is contained in the facet system of another d-polytope, then the two d-polytopes are combinatorially equivalent.

Let $\mathcal{V}(P)=\left\{x_{0}, x_{1}, \ldots, x_{n}\right\}, n \geq d$. We let $x_{i}<x_{j}$ if and only if $i<j$, and call $x_{0}<x_{1}<\cdots<x_{n}$ a vertex array of $P$. Let $G \in \mathcal{F}_{i}(P)$, $1 \leq i \leq d-1$, and

$$
\mathcal{V}(G)=G \cap \mathcal{V}(P)=\left\{y_{0}, y_{1}, \ldots, y_{m}\right\}
$$

that is, each $y_{s}$ is some $x_{t}$. Then $y_{0}<y_{1}<\cdots<y_{m}$ is the vertex array of $G$ if it is the ordering induced by $x_{0}<x_{1}<\cdots<x_{n}$. Finally, $P$ with $x_{0}<x_{1}<\cdots<x_{n}$ is Gale (with respect to the vertex array) if $\mathcal{V}(F)$ is a Gale set for each $F \in \mathcal{F}(P)$. We recall from [8, 9] that $P$ is cyclic if it is simplicial, and Gale with respect to some vertex array.

From [2], $P$ is a multiplex if there is a vertex array, say, $x_{0}<x_{1}<\cdots<$ $x_{n}$ such that

$$
\mathcal{F}(P)=\left\{\left[x_{i-d+1}, \ldots, x_{i-1}, x_{i+1}, \ldots, x_{i+d-1}\right] \mid 0 \leq i \leq n\right\}
$$

under the convention: $x_{t}=x_{0}$ for $t \leq 0$ and $x_{t}=x_{n}$ for $t \geq n$. A $d$ multiplex is a natural generalization of a $d$-simplex. Next, $P$ is multiplicial if each facet of $P$ is a $(d-1)$-multiplex with respect to the ordering induced by a fixed vertex array of $P$. Finally, $P$ is ordinary if it is Gale and multiplicial with respect to some vertex array. We note from [3] that if $P$ is an ordinary $d$-polytope and $d \geq 4$ then there is a complete description of $\mathcal{F}(P)$; furthermore, if $d$ is even then $P$ is cyclic. 
From [4, $P$ is periodically-cyclic if there is a vertex array, say, $x_{0}<x_{1}<$ $\cdots<x_{n}$ and an integer $k, d+2 \leq k \leq n+1$, such that

- $\left[x_{i+1}, x_{i+2}, \ldots, x_{i+k}\right]$ is a cyclic $d$-polytope with the induced vertex array for $-1 \leq i \leq n-k$, and

- $\left[x_{i+1}, x_{i+2}, \ldots, x_{i+k}, x_{i+k+1}\right]$ is not cyclic for $-1 \leq i \leq n-k-1$.

The integer $k$ is the period of $P$. We note that if $k=n+1$, then $P$ is cyclic.

Let $P$ be a periodically-cyclic $d$-polytope with $x_{0}<x_{1}<\cdots<x_{n}$. Since the condition that $\left[x_{i+1}, x_{i+2}, \ldots, x_{i+k+1}\right]$ is not a cyclic $d$-polytope may be satisfied in numerous ways, it follows that $P$ may be one of many combinatorial types. This observation remains valid even under the added assumption that $P$ is Gale with $x_{0}<x_{1}<\cdots<x_{n}$; see, for example, the bicyclic 4-polytopes in 11] that are Gale and periodically-cyclic [6]. For $d>4$, we know at present one class of realizable periodically-cyclic $d$-polytopes [4].

Proposition 3 Let $d \geq 6$ be even and $k \geq d+2$. Let $P_{k-1}$ be a cyclic d-polytope in $\mathbf{R}^{d}$ with vertex array $x_{0}<x_{1}<\cdots<x_{k-1}$. Then there exist a sequence of points $x_{n} \in \mathbf{R}^{d}$ and a sequence of polytopes $P_{n}=\left[P_{n-1}, x_{n}\right]$ $(n \geq k)$ such that

- $x_{n} \in\left\langle x_{0}, x_{n-k+1}, x_{n-k+2}, x_{n-1}\right\rangle$;

- $x_{n}$ is beyond each $F \in \mathcal{F}\left(P_{n-1}\right)$ with the property that $F \cap\left[x_{0}, x_{n-k+1}, x_{n-1}\right]=$ $\left[x_{0}, x_{n-1}\right]$; and

- $x_{n}$ is beneath each $F \in \mathcal{F}\left(P_{n-1}\right)$ with the property that $\left[x_{0}, x_{n-k+1}, x_{n-k+2}, x_{n-1}\right] \notin$ $F$ and $F \cap\left[x_{0}, x_{n-k+1}, x_{n-1}\right] \neq\left[x_{0}, x_{n-1}\right]$.

Each polytope constructed in this manner is Gale and periodically-cyclic with respect to $x_{0}<x_{1}<\cdots<x_{n}$ and with period $k$.

In 4 there is an explicit combinatorial description, depending only on $k$ and $n$, of the facets of these polytopes. It is tedious but straightforward to check that these facets are of the following combinatorial type. 
Definition. Let $Q \subset \mathbf{R}^{e}$ be an $e$-polytope with $\mathcal{V}(Q)=\left\{y_{0}, y_{1}, \ldots, y_{m}\right\}$, $m \geq e \geq 3$. Then $Q$ is an $e$-braxtope if there is a vertex array, say, $y_{0}<y_{1}<$ $\cdots<y_{m}$ such that

$$
\mathcal{F}(Q)=\left\{T_{0}, T_{1}, \ldots, T_{m-e+1}, E_{2}, E_{3}, \ldots, E_{m}\right\}
$$

with

$$
T_{i}=\left[y_{i}, y_{i+1}, \ldots, y_{i+e-1}\right] \text { for } 0 \leq i \leq m-e+1,
$$

and

$$
E_{j}=\left[y_{0}, y_{j-e+2}, \ldots, y_{j-1}, y_{j+1}, \ldots, y_{j+e-2}\right] \text { for } 2 \leq j \leq m,
$$

under the convention that $y_{t}=y_{0}$ for $t \leq 0$ and $y_{t}=y_{m}$ for $t \geq m$. For the sake of completeness, an $e$-braxtope is an $e$-simplex for $0 \leq e \leq 2$.

It is clear that an $e$-braxtope with $e+1$ vertices is an $e$-simplex, and that braxtopes are generalizations of simplices.

Finally, a $d$-polytope $P$ is braxial if each facet of $P$ is a $(d-1)$-braxtope with respect to the ordering induced by a fixed vertex array of $P$. As noted above, the periodically-cyclic $d$-polytopes constructed via Proposition 3 are Gale and braxial. The following result from [1] enables us to prove that Gale and braxial $d$-polytopes are periodically-cyclic for $d \geq 5$.

Lemma 4 Let $Q$ be an e-braxtope with $y_{0}<y_{1}<\cdots<y_{m}, m \geq e \geq 3$. Then with the convention that $y_{t}=y_{0}$ for $t \leq 0$ and $y_{t}=y_{m}$ for $t \geq m$ :

a. $\left[y_{0}, y_{t}\right] \in \mathcal{E}(Q)$ for $1 \leq t \leq m$.

b. $\left[y_{1}, y_{t}\right] \in \mathcal{E}(Q)$ if and only if $t \in\{0,2,3, \ldots, e\}$.

c. $\left[y_{t}, y_{m}\right] \in \mathcal{E}(Q)$ if and only if $t \in\{0, m-e+1, \ldots, m-1\}$.

d. For $2 \leq s \leq m-1,\left[y_{s}, y_{t}\right] \in \mathcal{E}(Q)$ if and only if $t \in\{0, s-e+1, \ldots, s-$ $1, s+1, \ldots, s+e-1\}$.

e. $\left[y_{0}, y_{t}, y_{t+1}, y_{t+e-1}, y_{t+e}\right] \in \mathcal{F}_{3}(Q)$ for $1 \leq t \leq m-e$.

f. $\left\{y_{t}, y_{t+1}, \ldots, y_{t+e}\right]$ is an affinely independent set for $0 \leq t \leq m-e$.

In addition, $Q$ is braxial and if $m \geq e+1$ then $\left[y_{0}, y_{1}, \ldots, y_{m-1}\right]$ is an e-braxtope with $y_{0}<y_{1}<\cdots<y_{m-1}$. 


\section{Gale and braxial polytopes}

Henceforth, we assume that $P$ is a Gale and braxial $d$-polytope with respect to $x_{0}<x_{1}<\cdots<x_{n}, n \geq d+1$ and $d \geq 3$. We simplify our notation by assuming also that $F$ and $F^{\prime}$ always denote facets of $P$ with the following properties (see Section 2 with $e=d-1$ ):

$$
\begin{aligned}
& y_{0}<y_{1}<\cdots<y_{m} \text { is the induced vertex array of } F \text { and } \\
& \mathcal{F}_{d-2}(F)=\left\{T_{0}, T_{1}, \ldots, T_{m-d+2}, E_{2}, E_{3}, \ldots, E_{m}\right\}, m \geq d-1 . \\
& z_{0}<z_{1}<\cdots<z_{u} \text { is the induced vertex array of } F^{\prime} \text { and } \\
& \mathcal{F}_{d-2}\left(F^{\prime}\right)=\left\{T_{0}^{\prime}, T_{1}^{\prime}, \ldots, T_{u-d+2}^{\prime}, E_{2}^{\prime}, E_{3}^{\prime}, \ldots, E_{u}^{\prime}\right\}, u \geq d-1 .
\end{aligned}
$$

In the next two proofs we distinguish the facets of a braxtope by the number of vertices. In particular, $E_{3}$ and $E_{m-2}$ are the only facets of the $(d-1)$-braxtope $F$ having exactly $d$ vertices.

Lemma 5 Let $F \in \mathcal{F}(P)$ with $x_{0} \notin F$. Then $F$ is a $(d-1)$-simplex.

Proof: The statement is trivial for dimension three, since all 2-dimensional braxtopes are simplices. Assume now that $d \geq 5$. With reference to (3.1), we suppose that $m \geq d$ and seek a contradiction.

We note that $x_{0} \notin F$ and the Gale property yield that $\left(y_{0}, y_{1}, y_{2}, y_{3}\right)=$ $\left(x_{r-1}, x_{r}, x_{s}, x_{s+1}\right)$ for some $2 \leq r<s \leq n-1$. We consider

$$
E_{3}=\left[y_{0}, y_{1}, y_{2}, y_{4}, \ldots, y_{d}\right]=\left[x_{r-1}, x_{r}, x_{s}, y_{4}, \ldots, y_{d}\right]
$$

and $F^{\prime} \in \mathcal{F}(P)$ with $z_{0}<z_{1}<\cdots<z_{u}$ such that $E_{3}=F \cap F^{\prime}$. Since $f_{0}\left(E_{3}\right)=d$, it follows that $E_{3} \in\left\{E_{3}^{\prime}, E_{u-2}^{\prime}\right\}$. If $E_{3}=E_{3}^{\prime}$ then $x_{r-1}<x_{r}<$ $x_{s}<z_{3}<y_{4}<\cdots<y_{d}<\cdots$ is the vertex array of $F^{\prime}$, and $F^{\prime} \cap\left\{x_{0}, x_{s+1}\right\}=$ $\emptyset$. Since $x_{0}$ and $x_{s+1}$ are separated by exactly three vertices of $F^{\prime}$, we have a contradiction of the Gale property.

Let $E_{3}=E_{u-2}^{\prime}=\left[z_{0}, z_{u-d+1}, \ldots, z_{u-3}, z_{u-1}, z_{u}\right]$. We note that $z_{0}=$ $y_{0}=x_{r-1}$, and hence, $z_{u-d+1}=y_{1}=x_{r}=z_{1}$ and $x_{r-1}<x_{r}<x_{s}<y_{4}<$ $\cdots<y_{d-2}<z_{u-2}<y_{d-1}<y_{d}$ is the vertex array of $F^{\prime}$. Again, $x_{0}$ and $x_{s+1}$ are separated by exactly three vertices of $F^{\prime}$.

The dimension four case is handled in a similar way.

Theorem A Let $P$ be a Gale and braxial d-polytope with respect to $x_{0}<$ $x_{1}<\cdots<x_{n}$. Let $d \geq 3$ be odd. Then $P$ is a cyclic d-polytope with respect to $x_{0}<x_{1}<\cdots<x_{n}$. 
Proof: We note that it is sufficient to prove that $P$ is simplicial. This holds for dimension three because all 2-dimensional braxtopes are simplices. So assume $d \geq 5$, and let $F \in \mathcal{F}(P)$ with $y_{0}<y_{1}<\cdots<y_{m}$. We suppose that $m \geq d$, and seek a contradiction.

Since $m \geq d$, it follows from Lemma 4 that $x_{0}=y_{0}$. If $x_{n}=y_{m}$ then $T_{1}=\left[y_{1}, \ldots, y_{d-1}\right]=F \cap \tilde{F}$ for some $\tilde{F} \in \mathcal{F}(P), \tilde{F} \cap\left\{x_{0}, x_{n}\right\}=\emptyset$ and $\tilde{F}$ is a $(d-1)$-simplex by Lemma 5 . Since $d$ is odd and $x_{0}$ and $x_{n}$ are separated by the $d$ vertices of $F^{\prime}$, it follows that $x_{n} \neq y_{m}$.

Since $x_{n} \notin F^{\prime}$, we obtain from the Gale property that

$$
\left\{y_{m-d}, y_{m-d+1}, \ldots, y_{m-1}, y_{m}\right\} \text { is a paired set }
$$

and $\left\{y_{m-3}, y_{m-2}, y_{m-1}, y_{m}\right\}=\left\{x_{r-1}, x_{r}, x_{s}, x_{s+1}\right\}$ for some $2 \leq r<s \leq$ $n-2$. We consider

$$
\begin{aligned}
E_{m-2} & =\left[y_{0}, y_{m-d+1}, \ldots, y_{m-3}, y_{m-1}, y_{m}\right] \\
& =\left[x_{0}, y_{m-d+1}, \ldots, y_{m-4}, x_{r-1}, x_{s}, x_{s+1}\right]
\end{aligned}
$$

and $F^{\prime} \in \mathcal{F}(P)$ with $z_{0}<z_{1}<\cdots<z_{u}$ such that $E_{m-2}=F \cap F^{\prime}$. Since $f_{0}\left(E_{m-2}\right)=d$, it follows that $E_{m-2} \in\left\{E_{3}^{\prime}, E_{u-2}^{\prime}\right\}$. If $E_{m-2}=E_{3}^{\prime}$ then $z_{0}=$ $y_{0}=x_{0}, z_{1}=y_{m-d+1}$ and $x_{0}<y_{m-d+1}<y_{m-d+2}<z_{3}<y_{m-d+3}<\cdots<$ $y_{m-4}<x_{r-1}<x_{s}<x_{s+1}<\cdots$ is the vertex array of $F^{\prime}$. If $E_{m-2}=E_{u-2}^{\prime}$ then $z_{0}=y_{0}<\cdots<y_{m-d+1}<\cdots<y_{m-4}<x_{r-1}<z_{u-2}<x_{s}<x_{s+1}$ is the vertex array of $F^{\prime}$. In case of the former, we note that $y_{m-d+2}$ and $y_{m-d+3}$ are not successive vertices, a contradiction by (3.3). In case of the latter, $F^{\prime} \cap\left\{x_{r}, x_{n}\right\}=\emptyset$ and $x_{r}$ and $x_{n}$ are separated by exactly three vertices of $F^{\prime}$.

In view of Theorem $\mathrm{A}$, we may now assume that $d \geq 4$ and even. Our first task is to determine $\mathcal{E}(P)$, and we let

$$
\mathcal{V}_{0}=\mathcal{V}_{0}(P)=\left\{x_{j} \in \mathcal{V}(P) \mid\left[x_{0}, x_{j}\right] \in \mathcal{E}(P)\right\}
$$

and

$$
\mathcal{V}_{i}=\mathcal{V}_{i}(P)=\left\{x_{j} \in \mathcal{V}(P) \mid x_{j} \neq x_{0} \text { and }\left[x_{i}, x_{j}\right] \in \mathcal{E}(P)\right\}, 1 \leq i \leq n
$$

Lemma $6 \mathcal{V}_{0}(P)=\mathcal{V}(P) \backslash\left\{x_{0}\right\}$

Proof: We note that $x_{j} \in \mathcal{V}_{0}$ for some $2 \leq j \leq n-1$, and that it is sufficient to show that $\left\{x_{j-1}, x_{j+1}\right\} \subset \mathcal{V}_{0}$. 
Since $\left[x_{0}, x_{j}\right] \in \mathcal{E}(P)$, there are $F^{*}$ and $\tilde{F}$ in $\mathcal{F}(P)$ such that $\left[x_{0}, x_{j}\right] \subseteq$ $F^{*} \cap \tilde{F}, x_{j-1} \notin F^{*}$ and $x_{j+1} \notin \tilde{F}$. Then $x_{j+1} \in F^{*}$ and $x_{j-1} \in \tilde{F}$ by the Gale property, and $\left\{x_{j-1}, x_{j+1}\right\} \subset \mathcal{V}_{0}$ by Lemma 4 (a)

Lemma 7 Let $1 \leq p<q<r \leq n$ and $\left[x_{p}, x_{r}\right] \in \mathcal{E}(P)$. Then $x_{q} \in$ $\mathcal{V}_{p}(P) \cap \mathcal{V}_{r}(P)$.

Proof: We note that it is sufficient to prove that $\left\{\left[x_{p}, x_{r-1}\right],\left[x_{p+1}, x_{r}\right]\right\} \subset$ $\mathcal{E}(P)$.

Since $\left[x_{p}, x_{r}\right] \in \mathcal{E}(P)$ and $x_{p} \neq x_{0}$, there is an $F \in \mathcal{F}(P)$ such that $\left[x_{p}, x_{r}\right] \subset F$ and $x_{p-1} \notin F$. Then $x_{p+1} \in F$ by the Gale property with, say, $x_{r}=y_{s}$ and $\left(x_{p}, x_{p+1}\right)=\left(y_{t}, y_{t+1}\right)$; see (3.1). Now either $F$ is a simplex and $\left[x_{p+1}, x_{r}\right] \in \mathcal{E}(P)$ or $x_{0}=y_{0}$ by Lemma [5. Let $x_{0}=y_{0}$. Then $y_{0} \neq y_{t}$ and we apply Lemma $4\left(\mathbf{c}\right.$ d) . Specifically, $\left[y_{t}, y_{s}\right]=\left[x_{p}, x_{r}\right] \in \mathcal{E}(P)$ implies that $s-d+2 \leq t \leq s-2$, whence $\left[x_{p+1}, x_{r}\right]=\left[y_{t+1}, y_{s}\right] \in \mathcal{E}(P)$.

In the case that $x_{r} \neq x_{n}$, a similar argument yields that $\left[x_{p}, x_{r-1}\right] \in$ $\mathcal{E}(P)$.

Let $\left[x_{p}, x_{n}\right] \in \mathcal{E}(P)$. We note that if $\left[x_{\ell}, x_{n}\right] \in \mathcal{E}(P)$ implies that $\left[x_{\ell}, x_{n-1}\right] \in \mathcal{E}(P)$ for some $1 \leq \ell<p$, then $\left[x_{\ell}, x_{n}\right] \in \mathcal{E}(P)$ implies $\left[x_{p}, x_{n-1}\right] \in$ $\mathcal{E}(P)$ by the preceding. Hence, we may assume that $p$ is the least positive integer such that $\left[x_{p}, x_{n}\right] \in \mathcal{E}(P)$. Then Lemma 4 (c) yields that any facet of $P$ that contains $\left\{x_{p}, x_{n}\right\}$ also contains vertices between $x_{p}$ and $x_{n}$. Let $x_{k}$ be the greatest of these vertices, and let $F \in \mathcal{F}(P)$ such that $\left\{x_{p}, x_{k}, x_{n}\right\} \subset F$. We claim that $\left[x_{p}, x_{k}\right] \in \mathcal{E}(P)$ and $x_{k}=x_{n-1}$.

We note that $($ see $(3.1))\left(x_{k}, x_{n}\right)=\left(y_{m-1}, y_{m}\right), x_{p} \in\left\{y_{0}, y_{m-d+2}\right\}$ by Lemma $4($ (c) $)$, and $\left[x_{p}, x_{k}\right] \in \mathcal{E}(P)$ by Lemma 4 (ad) and (dd). Since

$$
E_{m-2}=\left[y_{0}, y_{m-d+1}, \ldots, y_{m-3}, x_{k}, x_{n}\right]
$$

it is clear that if $x_{k} \neq x_{n-1}$, then $y_{m-2}=x_{k-1}$, and there is an $F^{\prime} \in \mathcal{F}(P)$ such that $E_{m-2}=F \cap F^{\prime}$ and $\left\{x_{p}, x_{k+1}, x_{n}\right\} \subset F^{\prime}$, a contradiction. Hence $x_{k}=x_{n-1}$.

Corollary 7.1 $\mathcal{V}_{1}(P)=\left\{x_{2}, \ldots, x_{r}\right\}$ and $\mathcal{V}_{n}(P)=\left\{x_{s} \ldots, x_{n-1}\right\}$ for some $d \leq r \leq n$ and $1 \leq s \leq n-d+1$.

We are now in the position to determine some of the subpolytopes of $P$. 
Lemma 8 Let $1 \leq s \leq n-d+1, \mathcal{V}_{n}(P)=\left\{x_{s}, x_{s+1}, \ldots, x_{n-1}\right\}$ and $S_{d} \subset$ $\left\{x_{s}, \ldots, x_{n-1}, x_{n}\right\}$. Then
a. $\left[S_{d}\right] \subset \mathcal{F}(P)$ and
b. $\left[x_{0}, x_{s-1}, x_{s}, x_{n-1}, x_{n}\right] \in \mathcal{F}_{3}(P)$.

Proof: (a) Since there is an $F^{\prime} \in \mathcal{F}(P)$ such that $x_{n} \in F^{\prime}$ and $x_{0} \notin F^{\prime}$, it follows that $F^{\prime}=\left[S_{d}^{\prime}\right]$ for some $S_{d}^{\prime} \subset\left\{x_{s}, \ldots, x_{n}\right\}$ by Lemma 5 and the Gale property. Since $s=n-d+1$ implies that $S_{d}^{\prime}=\left\{x_{s}, \ldots, x_{n}\right\}$, we may assume that $s \leq n-d$.

Let $S_{d} \subset\left\{x_{s}, \ldots, x_{n-1}\right\}$ such that $\left|S_{d} \cap S_{d}^{\prime}\right|=d-1$. Then $G=\left[S_{d} \cap S_{d}^{\prime}\right] \in$ $\mathcal{F}_{d-2}(P)$ and $G=F \cap\left[S_{d}^{\prime}\right]$ for some $F \in \mathcal{F}(P)$. By the Gale property, $S_{d} \subset$ $F$. Let $x_{t} \in S_{d} \subset\left\{x_{t}, \ldots, x_{n}\right\}$. Since $\left[x_{t}, x_{j}\right] \in \mathcal{E}(P)$ for each $x_{j} \in S_{d} \backslash\left\{x_{t}\right\}$, it follows from Lemma 4 that $x_{t}$ is the initial vertex of $F$. Thus, $x_{0} \notin F$ and $F=\left[S_{d}\right]$ by Lemma 5 .

If $s=n-d$ then we are done, and if $s<n-d$ then iterations of the preceding argument yield (a) .

(b) Let $S_{d-4} \subseteq\left\{x_{s+2}, \ldots, x_{n-2}\right\}$ and $S_{d}=\left\{x_{s}, x_{s+1}\right\} \cup S_{d-4} \cup\left\{x_{n-1}, x_{n}\right\}$. By (柆) and the Gale property,

$$
\left[x_{s}, S_{d-4}, x_{n-1}, x_{n}\right]=\left[S_{d}\right] \cap F
$$

for some $F \in \mathcal{F}(P)$ with $x_{s-1} \in F$. We note that $\left[x_{s-1}, x_{n}\right] \notin \mathcal{E}(P)$ implies that $F$ is not a simplex, and Lemma 5 implies that $x_{0} \in F$. Hence, $x_{0}=y_{0}$, $\left(x_{n-1}, x_{n}\right)=\left(y_{m-1}, y_{m}\right)$ and $\left(x_{s-1}, x_{s}\right)=\left(y_{m-d+1}, y_{m-d+2}\right)$ by Lemma 4 (c) $)$. Finally, Lemma $4($ (e) with $t=m-d+1$ yields the assertion.

Lemma 9 Let $2 \leq s \leq n-d+1$ and $\mathcal{V}_{n}=\left\{x_{s}, \ldots, x_{n-1}\right\}$. Then $\mathcal{V}_{n-1}(P)=$ $\left\{x_{s-1}, x_{s}, \ldots, x_{n-2}, x_{n}\right\}$.

Proof: By Lemmas[7and $8,\left\{x_{s}, \ldots, x_{n-2}, x_{n}\right\} \subset \mathcal{V}_{n-1}$ and $\left[x_{0}, x_{s-1}, x_{s}, x_{n-1}, x_{n}\right] \in$ $\mathcal{F}_{3}(P)$. The latter and $\left[x_{s-1}, x_{n}\right] \notin \mathcal{E}(P)$ yield that $\left[x_{s-1}, x_{n-1}\right] \in \mathcal{E}(P)$. Thus, it remains to show that $\left[x_{s-2}, x_{n-1}\right] \notin \mathcal{E}(P)$ for $s \geq 3$. We note that $\left[x_{s-1}, x_{n-1}\right] \in \mathcal{E}(P)$ yields that there is an $F \in \mathcal{F}(P)$ such that $\left[x_{s-1}, x_{n-1}\right] \subset F, x_{s} \notin F$ and $x_{s-2} \in F$.

If $x_{n} \in F$ then $\left[x_{s-1}, x_{n}\right] \notin \mathcal{E}(P)$ implies that $F$ is not a simplex and $y_{0}=$ $x_{0}<x_{s-2}$. We note that $\left(y_{m-1}, y_{m}\right)=\left(x_{n-1}, x_{n}\right)$. Thus $x_{s-1} \in \mathcal{V}_{n-1} \backslash \mathcal{V}_{n}$ and Lemma 4 (c)d yield that $y_{m-d}<x_{s-1}<y_{m-d+2}$. Then $x_{s-1}=y_{m-d+1}$, $x_{s-2}=y_{m-d}$ and $\left[x_{s-2}, x_{n-1}\right]=\left[y_{m-d}, y_{m-1}\right] \notin \mathcal{E}(P)$ by Lemma a(d). 
We may now assume that no facet of $P$ contains $\left\{x_{s-2}, x_{s-1}, x_{n-1}, x_{n}\right\}$. Clearly, this assumption and the Gale property yield that $\left[x_{s-2}, x_{s-1}, x_{n-1}\right] \notin$ $\mathcal{F}_{2}(P)$. Thus, $\left[x_{s-2}, x_{s-1}, x_{n-1}\right] \subset F$ implies that $x_{n-1}=y_{m}$ and $x_{s-2} \notin$ $T_{m-d+2}=\left[y_{m-d+2}, \ldots, y_{m}\right]$. Finally, $\left[x_{s-2}, x_{n-1}\right]=\left[y_{t}, y_{m}\right]$ for some $0<$ $t<m-d+2$ and Lemma प(C) yield that $\left[x_{s-2}, x_{n-1}\right] \notin \mathcal{E}(P)$.

Theorem B Let $P$ be a Gale and braxial d-polytope with $x_{0}<x_{1}<\cdots<$ $x_{n}, n \geq d+1 \geq 5$ and $d$ even. Then $P^{\prime}=\left[x_{0}, x_{1}, \ldots, x_{n-1}\right]$ is a Gale and braxial d-polytope with $x_{0}<x_{1}<\cdots<x_{n-1}$.

Proof: Let $F^{\prime} \in \mathcal{F}\left(P^{\prime}\right)$. We need to show that $F^{\prime}$ is a $(d-1)$-braxtope and that $\mathcal{V}\left(F^{\prime}\right)$ is a Gale set. We note that either $F^{\prime} \in \mathcal{F}(P)$, or $\left[F^{\prime}, x_{n}\right] \in \mathcal{F}(P)$, or $F^{\prime} \notin \mathcal{F}(P)$ and $x_{n}$ is beyond $F^{\prime}$. If $F^{\prime} \in \mathcal{F}(P)$ then $x_{n} \notin F^{\prime}$ and $F^{\prime}$ is braxial and $\mathcal{V}\left(F^{\prime}\right)$ is a Gale subset of $\mathcal{V}\left(P^{\prime}\right)$. If $F^{\prime} \notin \mathcal{F}(P)$ and $\left[F^{\prime}, x_{n}\right] \in$ $\mathcal{F}(P)$ then $\mathcal{V}\left(F^{\prime}\right)$ is necessarily a Gale set and $F^{\prime}$ is a $(d-1)$-braxtope by Lemma 4 ,

Let $F^{\prime} \notin \mathcal{F}(P)$ and $x_{n}$ be beyond $F^{\prime}$. We recall that $\mathcal{V}_{n}=\left\{x_{s}, \ldots, x_{n-1}\right\}$ for some $1 \leq s \leq n-d+1$, and note that $\mathcal{V}\left(F^{\prime}\right) \subset \mathcal{V}(P)$ and Lemma 1(c) yield that $\mathcal{V}\left(F^{\prime}\right) \subset \mathcal{V}_{n} \cup\left\{x_{0}\right\}$. Thus, if $s=n-d+1$ then $F^{\prime}=\left[x_{0}, x_{n-d+1}, \ldots, x_{n-1}\right]$, whence $F^{\prime}$ is a $(d-1)$-simplex and $\mathcal{V}\left(F^{\prime}\right)$ is a Gale set.

Let $s \leq n-d$ and $Y=\mathcal{V}\left(F^{\prime}\right) \cap\left\{x_{s}, \ldots, x_{n-2}\right\}$. We note that $|Y| \geq$ $d-2$, and claim that $Y$ is a paired set. We suppose otherwise and seek a contradiction. Since $\left[S_{d}\right] \in \mathcal{F}(P)$ for each $S_{d} \subset\left\{x_{s}, \ldots, x_{n-2}\right\}$ from Lemma 8, it follows that for some $t$ such that $1 \leq t \leq d / 2$ there is a maximal paired subset $S_{d-2 t}$ of $Y$ and a $t$-element subset $X_{t}$ of $Y \backslash S_{d-2 t}$ such that no two vertices of $X_{t}$ are successive.

Since $S_{d-2 t} \cup X_{t} \subset\left\{x_{s}, \ldots, x_{n-2}\right\}$ and $n \geq s+d$, there is an $S_{d} \subset$ $\left\{x_{s}, \ldots, x_{n-2}, x_{n-1}\right\}$ such that $S_{d-2} \cup X_{t} \subset S_{d}$. Since $\left[S_{d}\right] \in \mathcal{F}(P)$ is a simplex and $x_{n}$ is beneath $\left[S_{d}\right]$, it follows from Lemma 1(b) that

$$
G=\left[S_{d-2 t}, X_{t}, x_{n}\right] \in \mathcal{F}_{d-t}(P) .
$$

Let $F \in \mathcal{F}(P)$ such that $G \subseteq F$. Since $S_{d-2 t} \cup X_{t} \subset\left\{x_{s}, \ldots, x_{n-2}\right\}$ and $\mathcal{V}(F)$ is a Gale set, we obtain that there is a paired set

$$
S \subset \mathcal{V}(F) \cap\left\{x_{s-1}, x_{s}, \ldots, x_{n-2}, x_{n-1}\right\}
$$

such that $S_{d-2 t} \cup X_{t} \subset S$. We note that $\left|S_{d-2 t} \cup X_{t}\right|=d-t$ implies that $|S| \geq d$, and thus, $F$ is not a simplex and $\left|S \backslash\left\{x_{s-1}\right\}\right| \geq d-1$. Since $\left[x_{j}, x_{n}\right] \in \mathcal{E}(P)$ for $x_{j} \in\left(S \backslash\left\{x_{s-1}\right\}\right) \cup\left\{x_{0}\right\}$ and $x_{0} \in F$ by Lemma 5 . 
the contradiction we obtain is that $x_{n}$ is not a simple vertex of $F$, see Lemma $4(\mathrm{C})$.

In summary: $\mathcal{V}\left(F^{\prime}\right) \subset \mathcal{V}_{n} \cup\left\{x_{0}\right\}, Y=\mathcal{V}\left(F^{\prime}\right) \cap\left(\mathcal{V}_{n} \backslash\left\{x_{n-1}\right\}\right)$ is a paired set, $|Y| \geq d-2$ and $Y$ contains a paired set of cardinality at most $d-2$ by Lemma 8. Hence, $Y=S_{d-2}$ and $\mathcal{V}\left(F^{\prime}\right)=\left\{x_{0}\right\} \cup S_{d-2} \cup\left\{x_{n-1}\right\}$ is Gale with respect to $x_{0}<x_{1}<\cdots<x_{n-1}$.

Corollary B.1 Let $2 \leq s \leq n-d+1$ and $\mathcal{V}_{n}(P)=\left\{x_{s}, \ldots, x_{n-1}\right\}$. Then $\mathcal{V}_{n-1}\left(P^{\prime}\right)=\left\{x_{s-1}, x_{s}, \ldots, x_{n-2}\right\}$

Proof: In view of Corollary [7.1 and Lemma 9] it remains to show that $x_{s-2} \notin \mathcal{V}_{n-1}\left(P^{\prime}\right)$ for $s \geq 3$.

Let $\left[x_{s-2}, x_{n-1}\right] \in \mathcal{E}\left(P^{\prime}\right)$. Then $\left[x_{s-2}, x_{n-1}\right] \notin \mathcal{E}(P),\left[x_{s-2}, x_{n-1}, x_{n}\right] \notin$ $\mathcal{F}_{2}(P)$ and Lemma 1 yield that $x_{n}$ is beyond each $F^{\prime} \in \mathcal{F}\left(P^{\prime}\right)$ that contains $\left[x_{s-2}, x_{n-1}\right]$. Next, $\left[x_{s-2}, x_{n-1}\right] \in \mathcal{E}\left(P^{\prime}\right)$ and the Gale property of $P^{\prime}$ imply that there is an $F^{\prime} \in \mathcal{F}\left(P^{\prime}\right)$ such that $\left[x_{s-2}, x_{s-1}, x_{n-1}\right] \subset F^{\prime}$. Since $\left[x_{s-1}, x_{n-1}\right] \in \mathcal{E}(P)$ and $x_{n}$ is beyond $F^{\prime}$, it follows by Lemma 1 that $\left[x_{s-1}, x_{n-1}, x_{n}\right] \in \mathcal{F}_{2}(P)$ and $x_{s-1} \in \mathcal{V}_{n}(P)$, a contradiction.

Theorem C Let $P$ be a Gale and braxial d-polytope with $x_{0}<x_{1}<\cdots<$ $x_{n}, n \geq d+1 \geq 5$ and $d$ even. Let $P^{\prime}=\left[x_{0}, x_{1}, \ldots, x_{n-1}\right], F^{\prime} \in \mathcal{F}\left(P^{\prime}\right)$, and $\mathcal{V}_{n}(P)=\left\{x_{s}, \ldots, x_{n-1}\right\}, 2 \leq s \leq n-d+1$. Then

- $x_{n} \in\left\langle F^{\prime}\right\rangle$ if $\left[x_{0}, x_{s-1}, x_{s}, x_{n-1}\right] \subset F^{\prime}$,

- $x_{n}$ is beyond $F^{\prime}$ if $F^{\prime} \cap\left[x_{0}, x_{s-1}, x_{n-1}\right]=\left[x_{0}, x_{n-1}\right]$, and

- $x_{n}$ is beneath $F^{\prime}$ if $\left[x_{0}, x_{s-1}, x_{s}, x_{n-1}\right] \not \subset F^{\prime}$ and $F^{\prime} \cap\left[x_{0}, x_{s-1}, x_{n-1}\right] \neq$ $\left[x_{0}, x_{n-1}\right]$.

Proof: If $\left[x_{0}, x_{s-1}, x_{s}, x_{n-1}\right] \subset F^{\prime}$ then Lemma 8(b) implies that $x_{n} \in$ $\left\langle F^{\prime}\right\rangle$. Suppose $F^{\prime} \cap\left[x_{0}, x_{s-1}, x_{n-1}\right]=\left[x_{0}, x_{n-1}\right]$. Then Lemma प4(c) and $\mathcal{V}_{n-1}\left(P^{\prime}\right)=\left\{x_{s-1}, \ldots, x_{n-2}\right\}$ yield that

$$
\left|\mathcal{V}\left(F^{\prime}\right) \cap\left\{x_{s}, \ldots, x_{n-1}\right\}\right|=d-1 .
$$

Now Lemma四(c) yields also that $\left[F^{\prime}, x_{n}\right] \notin \mathcal{F}(P)$; that is, $x_{n} \notin\left\langle F^{\prime}\right\rangle$. Finally, $\left\langle F^{\prime}\right\rangle \cap\left\{x_{s-1}, x_{n}\right\}=\emptyset$, the Gale property of $P$ and (3.4) yield that $F^{\prime} \notin \mathcal{F}(P)$. Hence, $x_{n}$ is beyond $F^{\prime}$. 
Now assume

$$
\left[x_{0}, x_{s-1}, x_{s}, x_{n-1}\right] \not \subset F^{\prime}
$$

and

$$
F^{\prime} \cap\left[x_{0}, x_{s-1}, x_{n-1}\right] \neq\left[x_{0}, x_{n-1}\right] .
$$

Thus, either $x_{0} \notin F^{\prime}$ or $F^{\prime} \cap\left\{x_{0}, x_{n-1}\right\}=\left\{x_{0}\right\}$ or $F^{\prime} \cap\left[x_{0}, x_{s-1}, x_{s}, x_{n-1}\right]=$ $\left[x_{0}, x_{s-1}, x_{n-1}\right]$.

We note that $\left[F^{\prime}, x_{n}\right]$ is not a $(d-1)$-simplex, and recall from Lemma 5 that if a facet of $P^{\prime}$ or $P$ does not contain $x_{0}$ then it is a simplex.

Let $x_{0} \notin F^{\prime}$. Then $x_{0} \notin\left[F^{\prime}, x_{n}\right]$ and it follows that $F^{\prime}$ is a simplex and $\left[F^{\prime}, x_{n}\right] \notin \mathcal{F}(P)$; that is, $x_{n} \notin\left\langle F^{\prime}\right\rangle$. Now if $\mathcal{V}\left(F^{\prime}\right) \subset \mathcal{V}_{n}$ then $F^{\prime} \in \mathcal{F}(P)$ by Lemma 8 (a) , and if $\mathcal{V}\left(F^{\prime}\right) \not \subset \mathcal{V}_{n}$ then $F^{\prime} \in \mathcal{F}(P)$ by Lemma 1(c and 国), so $x_{n}$ is beneath $F^{\prime}$.

Let $x_{0} \in F^{\prime}$. We suppose that $x_{n} \in\left\langle F^{\prime}\right\rangle$, and seek a contradiction.

Since $F^{\prime} \in \mathcal{F}\left(P^{\prime}\right)$, there is a greatest vertex $x_{p}$ of $F^{\prime}$ and $x_{p} \leq x_{n-1}$. If $x_{p}=x_{n-1}$ then $\left\{x_{0}, x_{s-1}, x_{n-1}, x_{n}\right\} \subset\left\langle F^{\prime}\right\rangle$ by (3.6),$x_{s} \in\left\langle F^{\prime}\right\rangle$ by Lemma 8(b), and we have a contradiction by (3.5). Let $x_{p}<x_{n-1}$. With reference to (3.2), we note that $\left(z_{0}, z_{u-1}, z_{u}\right)=\left(x_{0}, x_{p}, x_{n}\right)$ for the facet $\left[F^{\prime}, x_{n}\right]$ of $P$ and that $z_{0}<z_{1}<\cdots<z_{u-1}$ is the vertex array of $F^{\prime}$ as a facet of $P^{\prime}$. Next

$$
x_{n}=z_{u} \in\left\langle x_{0}, z_{u-d+1}, z_{u-d+2}, x_{p}\right\rangle
$$

by Lemma $4($ (e) $)$, and

$$
E_{u-2}^{\prime}=\left[z_{0}, z_{u-d+1}, \ldots, z_{u-3}, z_{u-1}\right]=F^{\prime} \cap F^{\prime \prime}
$$

for some $F^{\prime \prime} \in \mathcal{F}\left(P^{\prime}\right)$. We observe that $x_{n} \in\left\langle F^{\prime \prime}\right\rangle$ by (3.7), and that $z_{u-1}=x_{p}<x_{n-1}$ and the Gale property yield that $x_{p-1}=z_{u-2} \notin F^{\prime \prime}$ and $x_{p+1} \in F^{\prime \prime}$. Clearly, $x_{p+1}$ is the greatest vertex of $F^{\prime \prime}$ as a facet of $P^{\prime}$. Thus $\left[E_{u-2}^{\prime}, x_{n}\right]$ is a $(d-2)$-face of the facet $\left[F^{\prime \prime}, x_{n}\right]$ of $P$ with $d=(d-1)+1$ vertices. Since $x_{p+1}$ is not in $\left[E_{u-2}^{\prime}, x_{n}\right]$ and $x_{0}=z_{0}<z_{u-1}=x_{p}<x_{p+1}<$ $x_{n}$ in the $(d-1)$-braxtope $F^{\prime \prime}$, this is a contradiction.

In summary, $x_{0} \in F^{\prime}, x_{n} \notin\left\langle F^{\prime}\right\rangle$ and either $x_{n-1} \notin F^{\prime}$ or $\left\{x_{s-1}, x_{n-1}\right\} \subset$ $F^{\prime}$. If $x_{s-1} \in F^{\prime}$ then $\mathcal{V}\left(F^{\prime}\right) \not \subset \mathcal{V}_{n} \cup\left\{x_{0}\right\}$ and, as already noted, $F^{\prime} \in \mathcal{F}(P)$. Hence, we suppose that $x_{n-1} \notin F^{\prime}$ and $\mathcal{V}\left(F^{\prime}\right) \subset \mathcal{V}_{n} \cup\left\{x_{0}\right\}$. Then $\mathcal{V}\left(F^{\prime}\right) \subset$ $\mathcal{V}_{n-1}\left(P^{\prime}\right) \cup\left\{x_{0}\right\}$ and it follows from Lemmas 6 and 7 that any two vertices of $F^{\prime}$ determine an edge of $F^{\prime}$. Finally, Lemma 4 and the Gale property yield that $F^{\prime}$ is a simplex and $F^{\prime}=\left[S_{d}\right]$ for some $S_{d} \subset\left\{x_{0}\right\} \cup\left\{x_{s}, \ldots, x_{n-2}\right\}$. Since $s \geq 2$, we have a contradiction. 
Theorem D Let $P$ be a Gale and braxial d-polytope with $x_{0}<x_{1}<\cdots<$ $x_{n}, n \geq d+1 \geq 7$ and $d$ even. Then for some $1 \leq s \leq n-d+1$, $\left[x_{j}, x_{n}\right] \in \mathcal{E}(P)$ if and only if $j=0$ or $s \leq j \leq n-1$. Furthermore

- $P$ is cyclic with $x_{0}<x_{1}<\cdots<x_{n}$ if $s=1$,

- $P$ is periodically-cyclic with period $k=n-s+2$ if $2 \leq s \leq n-d$, and

- $P$ is a d-braxtope with $x_{0}<x_{1}<\cdots<x_{n}$ if $s=n-d+1$.

Proof: The first claim of the theorem is from Lemma 6 and Corollary 7.1 .

Suppose $s=1$. Then $\left[x_{i}, x_{j}\right] \in \mathcal{E}(P)$ for any $x_{i} \neq x_{j}$ by Lemmas $[6]$ and 7 From this and Lemma 4, it follows that each facet of $P$ is a simplex. Since $P$ is Gale and simplicial, it is a cyclic polytope.

Let $2 \leq s \leq n-d$ and $k=n-s+2$. We observe that repeated applications of Theorem $\mathrm{B}$ and its corollary yield that $\tilde{P}=\left[x_{0}, x_{1}, \ldots, x_{n-s+1}=x_{k-1}\right]$ is a Gale and braxial $d$-polytope with $k-1 \geq d+1$ and $\mathcal{V}_{k-1}(\tilde{P})=$ $\left\{x_{1}, \ldots, x_{k-2}\right\}$. Thus $\tilde{P}$ is cyclic, and it readily follows from Proposition 3 and Theorem $\mathrm{C}$ that $P$ is periodically-cyclic with period $k$.

Finally, let $s=n-d+1$ and $P_{r}=\left[x_{0}, x_{1}, \ldots, x_{r}\right], d \leq r \leq n$. We note that $P_{d}$ is a $d$-simplex, and thus, it is a $d$-braxtope. Next, $\mathcal{V}_{n}(P)=$ $\left\{x_{n-d+1}, \ldots, x_{n-1}\right\}$ and repeated applications of Theorem $\mathrm{B}$ and its corollary yield that $\mathcal{V}_{r}\left(P_{r}\right)=\left\{x_{r-d+1}, \ldots, x_{r-1}\right\}$ for $d+1 \leq r \leq n$. We recall that $x_{r}$ is beyond $F \in \mathcal{F}\left(P_{r-1}\right)$ only if $\mathcal{V}(F) \subset \mathcal{V}_{r}\left(P_{r}\right) \cup\left\{x_{0}\right\}$, and thus, $x_{r}$ is beyond only $\left[x_{0}, x_{r-d+1}, \ldots, x_{r-1}\right] \in \mathcal{F}\left(P_{r-1}\right)$. With the assumption that $P_{r-1}$ is a $d$-braxtope, it is now easy to check that Theorem $\mathrm{C}$ and the preceding yield that $P_{r}$ is a $d$-braxtope for $d<r \leq n$.

In fact, we have shown that the Gale and braxial polytopes in even dimension $d \geq 6$ are exactly the polytopes of Proposition 3, that is, the periodically-cyclic Gale polytopes constructed in [4. Henceforth call these polytopes Gale-braxial polytopes. In view of the comments preceding Proposition 3. we conjecture that, for even $d \geq 6$, there exist periodically-cyclic $d$-polytopes that are Gale, but not Gale-braxial.

Problem 1 Determine all periodically-cyclic $d$-polytopes for even $d \geq 6$.

In 44 it was shown that the construction of Proposition 3, applied in dimension four, produces polytopes that are not periodically-cyclic. Theorems $\mathrm{A}$ C show that Gale and braxial 4-polytopes are polytopes constructed in this manner, so they are not periodically-cyclic. As mentioned in Section 2, some bicyclic polytopes are both Gale and periodically-cyclic (and 
necessarily not braxial) [6. Naturally, we would like a better understanding of braxial polytopes with regard to these other properties.

Problem 2 Determine the explicit facet structure of Gale-braxial 4-polytopes.

Problem 3 Determine all 4-polytopes that are braxial and periodicallycyclic.

Of course, we can ask for a complete classification of braxial 4-polytopes but this is not likely to be tractable.

We have focused here on even-dimensional polytopes. In odd dimensions ordinary polytopes have been studied as a generalization of cyclic polytopes. The question of whether ordinary polytopes are periodically cyclic is open; Dinh [7] proved that ordinary polytopes satisfy a weaker condition, local neighborliness.

\section{References}

[1] M. M. Bayer and T. Bisztriczky. On braxtopes, a class of generalized simplices. arXiv:math.CO/0607396, 2006.

[2] T. Bisztriczky. On a class of generalized simplices. Mathematika, 43:274-285, 1996.

[3] T. Bisztriczky. Ordinary $(2 m+1)$-polytopes. Israel J. Math., 102:101$123,1997$.

[4] T. Bisztriczky. A construction for periodically-cyclic Gale $2 m$ polytopes. Beiträge Algebra Geom., 42(1):89-101, 2001.

[5] T. Bisztriczky and K. Böröczky. Oriented matroid rigidity of multiplices. Discrete Comput. Geom., 24(2-3):177-184, 2000. The Branko Grünbaum birthday issue.

[6] T. Bisztriczky and K. Böröczky. On periodically-cyclic Gale 4polytopes. Discrete Math. 241:103-118, 2001. Selected papers in honor of Helge Tverberg.

[7] T. Dinh. Ordinary Polytopes. Ph. D. Thesis, The University of Calgary, 1999.

[8] D. Gale. Neighborly and cyclic polytopes. In Proc. Sympos. Pure Math., Vol. VII, pages 225-232. Amer. Math. Soc., Providence, R.I., 1963. 
[9] B. Grünbaum. Convex polytopes, volume 221 of Graduate Texts in Mathematics. Springer-Verlag, New York, second edition, 2003. Prepared and with a preface by Volker Kaibel, Victor Klee and Günter M. Ziegler.

[10] V. Klee and G. J. Minty. How good is the simplex algorithm? In Inequalities, III (Proc. Third Sympos., Univ. California, Los Angeles, Calif., 1969; dedicated to the memory of Theodore S. Motzkin), pages 159-175. Academic Press, New York, 1972.

[11] Z. Smilansky. Bi-cyclic 4-polytopes. Israel J. Math., 70(1):82-92, 1990.

[12] G. Ziegler. Lectures on polytopes, volume 152 of Graduate Texts in Mathematics. Springer-Verlag, New York, 1995. 